\title{
Parkour y movimiento humano. Sentidos y significados de su práctica en Bogotá
}

\author{
Parkour and Human Movement; \\ Meanings and Significance of its Practice in Bogota
}

\author{
Jhon Carlos Cortés-Murillo (i) https://orcid.org/0000-0001-8160-7979 \\ Corporación Universitaria CENDA, Colombia, jhon.cortes@cenda.edu.co \\ Ita del Pilar Perea-Baena (iD https://orcid.org/0000-0002- 4496-9863 \\ Corporación Universitaria CENDA, Colombia, ita.perea@cenda.edu.co \\ Johan Sarmiento-Rojas \\ Corporación Universitaria CENDA, Colombia johan.sarmiento@cenda.edu.co
}

Recepción:

06/02/2018

Aprobación:

$20 / 05 / 2018$

\begin{abstract}
This study aims to interpret the experiences and meanings that traceurs - practitioners of parkour - configure in their relationship with the public spaces of the city of Bogota. This analysis is located in the perspective of human kinetics, taking the intentional movement as a central category. It is an interpretive and phenomenological study about the relationship between subject-space through movement-experience. This study used semistructured individual interviews, as well as group discussion interviews, with the aim to understand the subjective characteristics and collective sense that defines such practice. The findings show creativity, adaptability and ecology of movement as fundamental characteristics of the activity, in which movements require a greater degree of delicacy to harmonize with and preserve the spaces of the city.
\end{abstract}

Key words: parkour, mobility, intentional movement, body.

Resumen: Este estudio tiene por objetivo interpretar las experiencias y significados que los traceurs - practicantes de parkour- configuran en su relación con los espacios públicos de la ciudad de Bogotá. Este análisis está situado en la perspectiva de la motricidad humana y retoma el movimiento intencionado como categoría central. Metodológicamente es un estudio fenomenológico abocado a la relación sujeto-espacio a través del movimiento y la experiencia, razón por la cual recurre a entrevistas semiestructuradas individuales y entrevistas grupales de discusión, con el fin de interpretar las particularidades del mundo subjetivo y del sentido colectivo que define la práctica. Los hallazgos muestran la creatividad, la adaptabilidad y la ecología del movimiento como características fundamentales de la práctica en la que los movimientos requieren mayor grado de delicadeza para armonizar con los obstáculos y preservar los espacios de la ciudad.

Palabras clave: parkour, motricidad, movimiento intencionado, cuerpo. 


\section{Introducción}

Bogotá, como cualquier ciudad del mundo, se configura como escenario multicultural y diverso. Sus espacios dan lugar a innumerables formas de expresión que la recrean cotidianamente. Entre dichas expresiones se encuentra el parkour $(\mathrm{PK})$, una práctica corporal alternativa que reclama un lugar en la vida de la ciudad, caracterizada porque sus practicantes, denominados traceurs, vocablo francés que traducido al español significa trazadores, superan obstáculos físicos -edificios, muros, sillas, cajones, entre otros-, mediante la versatilidad del movimiento humano y las destrezas motoras. Sus practicantes le denominan el arte del desplazamiento y afirman que su práctica se sustenta en la filosofía ser y durar (Cortés y Certuche, 2014).

En la actualidad parece que su crecimiento ha generado tensiones que confrontan las convenciones de uso de la infraestructura a partir de la relación espacio-prácticas-cuerpo. Así, la configuración de la ciudad y la subjetividad de sus habitantes se manifiesta en formas particulares de apropiar y construir el sentido del espacio en la cotidianidad, procesos que al pasar el tiempo se vuelven referentes sensibles para quienes participan de la vida urbana (Sennett, 1987). Con base en lo anterior, es importante señalar que el carácter valorativo y específico del uso de la infraestructura de la ciudad se encuentra delimitado por la historicidad, diversidad y significación de los escenarios.

Es un proceso de acciones colectivas e individuales que supera toda intención preconcebida; por ejemplo, las fronteras invisibles, aquellas en las que una esquina barrial cualquiera puede considerarse un límite territorial que al ser trasgredido genera reacciones violentas entre jóvenes de un lado y otro. Esta situación muestra que las valoraciones sobre el espacio van más allá de todo propósito funcional del diseño arquitectónico. En este sentido, el parkour y la apropiación temporal que sus practicantes hacen de los distintos lugares de la ciudad denota una ruptura frente al uso convencional del diseño, pues en los gestos de sus cuerpos se expresan otras formas de moverse y vivir el espacio.

En cuanto al conocimiento del parkour, autores como Viera y De Sousa (2016) examinan veinte estudios, sus perspectivas teóricas y metodológicas, destacando en cada uno de ellos los ejes de análisis entre los que mencionan las implicaciones de la practica en la sociedad, la personalidad y comportamiento frente al riesgo de los practicantes, la apropiación y reformulación del espacio urbano, las lesiones resultantes del entrenamiento imprudente, las emociones en la práctica, las cuestiones de la producción cinematográfica, 
las representaciones del parkour, la influencia de las tecnologías y los procesos deportivos, sin olvidar que la mayoría de los estudios se encuentran en el campo de la sociología. Los métodos utilizados son variables y contemplan investigaciones cualitativas, cuantitativas y revisiones bibliográficas.

Otros estudios, como los elaborados por Gutiérrez y Rosales (2011) y Redondo (2011) consideran que el parkour es básicamente una actividad en la que se utiliza distintos tipos de arquitectura urbana para seguir el camino, superando los obstáculos que se anteponen al practicante, razón por la cual requiere de una intencionalidad reflexiva sobre el cuerpo y la arquitectura urbana que los posiciona espacialmente. Así, la configuración de la práctica va más allá de la técnica y el desarrollo de habilidades, pues se trata de un estilo de vida que confronta la naturalización de la relación acción corporal-función espacial, postura a partir de la cual los traceurs cuestionan y resignifican el cuerpo y los espacios mediante movimientos cargados de sentido.

Para contextualizar lo anterior, Carvalho y Pereira (2008) analizan a un grupo de practicantes de parkour en Sao Paulo. Su propósito es comprender la práctica como subcultura; para los autores, el parkour ha construido su propia identidad, un carácter propio de resistencia social que se manifiesta sobre todo en la lucha por un espacio de expresión. En esta misma línea de análisis cultural, Scarnatto y Díaz (2010) elaboran algunas reflexiones tomando en cuenta la dialéctica cuerpo-cultura en el contexto urbano de dos ciudades argentinas. El presente trabajo coincide con el estudio realizado por Silva (2012), quien observa colectivos de jóvenes practicantes de parkour en Teresina (Brasil), en los que las identidades compartidas les hace visibles socialmente. En otro sentido, Ferro (2011) aporta elementos frente al origen, proyección e implicación de la práctica en el contexto urbano, en particular en la forma de habitar, recorrer y usar los espacios.

En cuanto al contexto bogotano, Rotawisky $(2013,2015)$ explora la experiencia incorporada. Busca comprender la forma en que sus practicantes producen subjetividades nómadas y cómo, a través de transformaciones corporales, crean heterotopías en las ciudades contemporáneas. La autora define al parkour como una disciplina que construye un cuerpo capaz de trazar trayectos en la ciudad (Rotawisky, 2013). Este repaso sobre los estudios asociados al parkour permiten identificar cierta confluencia de las perspectivas culturales y de grupo, razón por la cual la presente investigación reorienta la mirada y se pregunta por el carácter individual del practicante, sin perder de vista el tejido colectivo que allí se configura.

Por tanto, el interrogante que moviliza esta investigación es: ¿Cuáles son las experiencias y sentidos que los practicantes de parkour configuran en su 
relación con los espacios de la ciudad? Para ello la educación física ha planteado algunos debates y reflexiones en torno al cuerpo y su experiencia, donde se destaca la perspectiva fenomenológica de la ciencia de la Motricidad Humana (Sergio, 1996 y 1999), propuesta epistemológica que aboga por la comprensión y construcción del cuerpo a partir de la experiencia de las acciones, en especial aquellas que denotan sentido y significado mediante la intencionalidad del movimiento.

\section{La motricidad y el movimiento intencionado}

Para la ciencia de la Motricidad Humana, el movimiento va más allá de la mecánica, pues este se encuentra cargado de intencionalidad en virtud de su razón de ser, anidado en la vida cotidiana, la sociedad y la cultura. El concepto de cuerpo relacionado con lo únicamente funcional toma aquí una re-significación, es ahora un cuerpo significativo y significante, un cuerpo provisto de pensamiento, emociones y de movimiento que actúa como una totalidad en relación estrecha con el mundo (Benjumea, 2010).

La intencionalidad del movimiento implica la exaltación de lo humano como trascendencia y superación; frente a esto, es importante señalar que al cuerpo físico y dicotómico le pertenece el movimiento; sin embargo, una fenomenología corporal se opone a tal consideración, pues el cuerpo, más allá de la sustancia, es esencia. En este sentido, dicha esencia es construcción, formación, recordación, narración, expresión, comunicación, que a la luz de la fenomenología constituye la corporeidad, se trata de un cuerpo-experiencia al que le corresponde la intención, la voluntad, el deseo, el recuerdo, la imaginación, pues vivir este cuerpo significa "sentir, pensar, y querer [...] pienso y siento al tiempo que hago; actúo porque siento y pienso. El cuerpo humano no solo hace" (Trigo, 2000: 32); una concepción en la que coexiste el símbolo, la materia y el deseo. El movimiento intencionado va más allá de una expresión física, se trata de una forma de existencia humana contextualizada, razón por la cual se hace indispensable para comprender los sentidos anclados a las prácticas corporales.

En el movimiento intencionado es posible observar lo motriz, el pensamiento, la interacción social y lo valorativo que confluyen en el sujeto y su cuerpo, se trata de "un proyecto motor, una intencionalidad motriz [...] todo movimiento es indisolublemente movimiento y conciencia [...] son una totalidad única" (Ponty, 1994: 127). Sin embargo, esta perspectiva no pretende establecerse como hegemónica y sostenerse como única y desde la cual se deba comprender el movimiento humano; más bien trata de ampliar 
el horizonte de comprensión, de aclarar que el conocimiento del cuerpo y sus movimientos no se reduce a lo funcional, sino que es funcional-significativaexistencial (Martínez, 2009).

En la motricidad humana cabe la explicación funcional-orgánica, pero estudiar la intencionalidad de estos movimientos implica centrarse en la experiencia del sujeto y descubrir ese nudo de significación. Por tanto, toda explicación de la motricidad desde la perspectiva funcional-orgánica es incompleta; con ella se puede elucidar la mecánica causal, pero no la intencionalidad portadora de significados que se manifiesta en las expresiones del cuerpo. Para quien practica el futbol, la cancha es vivenciada de forma significativa, jugar en zona defensiva puede significar angustia; o jugar de guardameta puede significar esperanza, anhelo o ansiedad.

En consecuencia, el movimiento intencionado se dispone en un espacio que supera las configuraciones teórico-geométricas y lo sitúa en un marco de experiencia que involucra deseos, relaciones, comunicación y emoción, entre otros aspectos que definen la condición humana. Moverse es vivir, evocar, sentir, valorar, pero también es el recorrido del cuerpo en el tiempo y el espacio; el movimiento humano intencionado es signo, significante y significado; es una experiencia que va más allá de la función natural; el movimiento intencional es palabra y memoria, moverse es ser.

\section{El cuerpo conjugado}

Cuando se precisa reflexionar sobre lo humano, es decir, sobre las experiencias que nos constituyen razón, emoción y corporeidad, es fundamental reconocer el cuerpo vivido, sentido, recordado, recortado, modelado, figurado, no como estructura de soporte que denota presencia independiente de la subjetividad, sino como condición existencial articulada al mundo. En otras palabras, la existencia humana está configurada en el cuerpo; en sus experiencias, acciones y obras, en sus movimientos y desplazamientos, en las tensiones y acuerdos, en las decisiones y los actos,

que por medio de la instrumentalidad del cuerpo [...] se presenta y se representa como un ser expresivo y dramático que actúa sobre el escenario del gran teatro del mundo respondiendo, cotidianamente, a las preguntas, los retos y las novedades que se le presentan como consecuencia de su radical no finitud constitutiva (Goffman, 1989: 76).

La cotidianidad del cuerpo trabaja las formas particulares y subjetivas necesarias para enfrentar los obstáculos sensitivos y simbólicos, pero a la vez destaca la importancia del espacio construido como elemento estructurante 
de dichas relaciones; "es evidente que las relaciones espaciales de los cuerpos humanos determinan en buena medida la manera en que las personas reaccionan unas respecto a otras" (Sennett, 1987: 19); por ende, la interpelación sensitiva a través del movimiento va configurando los sentidos de los actos corporales; es decir, el cuerpo y las sustancias que lo soportan hacen parte de una relación constante que fluye, se mueve y comunica.

El cuerpo no es solamente órganos o sistemas que responden a condiciones ambientales; de acuerdo con Le Breton (2002: 6), "cada sociedad esboza, en el interior de su visión del mundo, un saber singular sobre el cuerpo: sus contribuciones, sus usos, sus correspondencias. Le otorga sentido y valor. Las concepciones del cuerpo son tributarias de las concepciones de las personas". En ellas se hace explícito un esquema de relaciones que constituyen sentidos sobre las acciones y los objetos, lo cual significa apelar en primera instancia al lenguaje común que los gesta como clave de entendimiento; y en segunda instancia, atender al llamado del ser corporeizado, pues la intencionalidad del movimiento humano trasciende los esquemas biomecánicos, según Trigo (2000).

El Humanes ya no sólo posee un cuerpo que sólo hace, sino que su existencia es corporeidad, que implica hablar de su integralidad y no de una parte del ser, esa persona que vive, siente, piensa, hace cosas, se desplaza, se crece, se emociona, se relaciona con otros y con el mundo que le rodea (Trigo, 2000: 7).

Frente a lo anterior, se puede afirmar que el cuerpo es comunicación, medio y acción de socialización, es expresión, sentimiento y emoción; de acuerdo con McLaren (1997: 90), existe un proceso de apropiación corporizado denominado encarnación: "ser encarnado no es solo apropiarse de símbolos, sino también identificarse con el símbolo del que uno se apropia, es decir [...] alcanzar una correspondencia entre [...] el discurso y el sujeto". Por consiguiente, en lo corporal se configura la identidad que trasciende como forma de vida que no es otra cosa que el reconocimiento de la condición humana expresada en las acciones del ser en y con el mundo. El hacer, sentir, pensar y querer de la persona, lo esencial humano son y viven en su corporeidad.

Corporeidad es tener conciencia de nuestro cuerpo. Supone el hecho de encontrarnos y enfrentarnos con el mundo, con el otro, con un régimen de sentido y un modo de construir, a partir de regularidades heredadas y asumida nuestra propia realidad. Tomar conciencia de nuestro cuerpo no es solo la experiencia de lo que sentimos a través de él, sino el conjunto de significaciones que a partir de él atribuimos al mundo, insertos en un juego de reconocimiento recíproco con los otros (Moreno, 2009: 152). 
La corporeidad se estructura en aquello que Mèlich (1994: 79) denomina el ser corpóreo, cuyo sentido está en "abrirse a toda una serie de dimensiones antropológicas y sociales, significa ser-sí-mismo, pero también ser-tú, ser-con y ser-en el mundo [...] Pero no ser en el mundo receptivo, paciente, sino básicamente ser con el mundo". En consecuencia, los sujetos existen en la medida que se apropian de las transiciones y experiencias que suceden en sus cuerpos; así comparten con los demás lo que Ponty (1994) denomina la intersección de las experiencias subjetivas e intersubjetivas, "condición de presencia, participación y significación del hombre en el mundo" (Trigo, 1999: 60). En esta perspectiva fenomenológica, la corporeidad es la forma y significado que adquiere el cuerpo, es la organización de las experiencias que se imprimen a través de acciones y movimientos.

La corporeidad significa estar en el mundo como expresión, vinculada al espacio y al tiempo; según Eugeni (1991: 16) "forman un conjunto dialéctico, ya que, difícilmente, podremos entender uno sin tener en cuenta al otro". Sin embargo, tal entendimiento es dependiente de la interacción del sujeto con sí mismo, con los demás, con el espacio-tiempo, pues mediante estos vínculos se hace evidente su existencia. Así, el movimiento deja de ser una manifestación natural de fuerzas y trasciende hacia la intención, proceso de interacción entre la conciencia, la corporeidad y el entorno representado en los sentidos, en las finalidades, en los deseos.

La intención o deseo de movimiento constituye modelos de expresión que comunican, enuncian y significan la acción corporal; según Maturana (1996), la calidad de interacción de estas dimensiones en la acción dependerá de la intención de quien se mueve en relación con sus procesos orgánicos y su contexto sociocultural, pero entre todos hay una interdependencia que no se puede menospreciar y que sólo tiene sentido separarlas para hablar de ellas. Cada sujeto, cada corporeidad denota una intencionalidad; aspecto que, según López (2008), obedece al conocimiento que tenemos de nosotros mismos y del mundo exterior, es el rasgo distintivo de la vida mental, lo cual significa que las acciones humanas no están aisladas de su entorno de producción ni de las formas de interpretación subjetiva.

\section{Diseño metodológico}

Esta es una investigación fenomenológica que interpreta las experiencias y significados que los traceurs asignan al parkour y su relación con la ciudad. En este sentido, la observación recae sobre la cotidianidad espacializada que le da sentido a la práctica. Para ello, el abordaje se plantea desde la perspectiva de la 
Motricidad Humana elaborada por Sergio (1999) y la comprensión metodológica de la fenomenología de Moreira (1998) y Leal (1997). La recolección de la información se efectuó mediante entrevistas grupales de discusión y entrevistas individuales semiestructuradas, que permiten contrastar el mundo colectivo y subjetivo. Para las entrevistas se diseñaron dos guiones de preguntas y dos protocolos de aplicación, cuyo contenido y forma fueron validados por tres expertos en investigación cualitativa y una prueba piloto.

\section{Los participantes}

Los participantes pertenecen a agrupaciones de parkour que hacen uso del espacio público al interior del perímetro urbano de la ciudad de Bogotá. Se trata de grupos que se reúnen periódicamente y presentan algún grado de organización y estructura formal o informal. Su vinculación se hizo mediante tres criterios que garantizan el desarrollo del trabajo: a) mostrar interés en participar de la investigación, b) que los participantes contaran con más de cinco años de práctica, c) realizar la práctica con frecuencia no menor a cuatro horas por semana.

Estos aspectos garantizan el vínculo permanente del sujeto con la práctica y, por ende, la existencia de una relación que constituye el carácter subjetivo que deriva en los significados asociados al parkour y a su relación con el uso y trasformación de los espacios comunes de la ciudad. Es importante señalar que por tratarse de una investigación fenomenológica esta se aparta de los criterios de representatividad estadística; la selección de los participantes se realizó considerando la relevancia de los casos y las expectativas generadas teóricamente para el trabajo de campo.

\section{Trabajo de campo}

En el trabajo de campo se llevaron a cabo cuatro entrevistas grupales de discusión que contemplaron tres encuentros por cada una de ellas, en las que participaban cinco practicantes escogidos por los integrantes de los grupos. Esto corresponde a doce sesiones de trabajo con un promedio de una hora y 17 minutos de grabación para cada sesión (véase Tabla $1^{1}$ ).

En cuanto a las entrevistas individuales, se realizaron 12; del mismo modo cada entrevista se constituye de tres encuentros con cada sujeto, para un total de 36 sesiones. En promedio cada encuentro tuvo una duración de

1 Las tablas se encuentran en el Anexo, al final del presente artículo (Nota del editor). 
una hora y 36 minutos registrados. Este esquema, que también se aplicó en el proceso de las entrevistas grupales de discusión, fue diseñado con el propósito de enfocar y ajustar paulatinamente la indagación. Para esto, la información registrada en cada encuentro fue sometida a lectura analítica o inferencial, lo cual permitía analizar de forma gradual la información recabada e identificar aspectos de interés que después dieron lugar a nuevos interrogantes resueltos en los encuentros consecuentes.

En cuanto a las edades y participantes de las entrevistas individuales, en cada grupo se escogió al traceur que contaba con el mayor número de años de experiencia: para el grupo A-P 4, grupo B-P 5, grupo C-P 1, grupo D-P 1, como se muestra en la Tabla 2. Buscando contar con un marco de información comparativo y amplio frente al sentido subjetivo de la práctica, el restante de entrevistados (8) corresponde a practicantes que gozan de reconocimiento y aprecio en la escena parkour de Bogotá y no pertenecen a los grupos entrevistados. Las entrevistas se realizaron una vez finalizado el trabajo con los grupos entre agosto de 2016 y febrero de 2017.

Todos los entrevistados -individuos y miembros de grupos- en esta investigación son hombres. Las edades y años de experiencia (AE) de los participantes $(\mathrm{P})$ de las entrevistas se distribuyen de la manera en que aparece en la Tabla 2.

Todos los participantes involucrados desde el inicio hasta el final del estudio completaron el proceso. Respecto a la captura de información, el registro fue hecho mediante grabadora de voz, previa autorización y firma de consentimiento informado y reserva de identidad de los entrevistados.

\section{Esquema de análisis}

Para la organización, tratamiento y análisis de la información es importante recalcar que la dimensión fenomenológica de análisis que convoca en este estudio es el espacio, es decir, la relación del traceur y sus movimientos con la ciudad y los lugares de práctica. Para ello se determinaron tres momentos: 1) corresponde a la codificación axial de las entrevistas, (Strauss y Corbin, 2002); el propósito de este paso es identificar en las transcripciones aquellas declaraciones asociadas a la dimensión sujeto-espacio; 2) en este momento se diseña una matriz de doble entrada en la cual se clasifica la información considerando la dimensión sujeto-espacio como caso, y las técnicas de recolección de información como variables; esta estructura permite identificar las diferencias entre las declaraciones individuales y colectivas, y a la vez facilita comprender la carga subjetiva, influencia y rasgos discursivos de los 
practicantes; 3) la información clasificada es sometida a codificación abierta (Coffey y Atkinson, 2003), con el fin de construir una red de sentidos a partir de la identificación y relación de elementos recurrentes, de los énfasis y disonancias discursivas, de los tipos de experiencia, y de los significados que los practicantes atribuyen al espacio y a las experiencias gestadas en la práctica.

Con estos elementos se genera una descripción teórica que expresa las relaciones que se presentan en el apartado de Resultados. Para validar la información se realizaron dos procesos, el primero consistió en seleccionar, agrupar y enviar a cada entrevistado un documento con todas las respuestas que suministró. Dicho documento fue estructurado a partir de tres elementos: 1) la pregunta que se hizo en la entrevista, 2) la respuesta del entrevistado, y 3) una casilla de observaciones sobre la información provista en caso de querer aclarar la respuesta.

El segundo proceso corresponde a la valoración de las descripciones teóricas por parte de los entrevistados. Para esto se hizo el envío de los textos descriptivos junto con un cuestionario de cinco preguntas en escala Likert, con el propósito de establecer el grado de conformidad entre los participantes de la investigación. Cabe destacar que para la identificación de las declaraciones o citas textuales de los entrevistados se usó el siguiente esquema y sus combinaciones: $\mathrm{EI}=$ entrevista individual, $\mathrm{EG}=$ entrevista grupal, $\mathrm{P}=$ participante $\mathrm{G}=$ grupo.

\section{Resultados}

El análisis devela distintas tensiones asociadas a la apropiación y uso de los lugares entre las que se destaca la percepción del espacio, las formas efímeras del habitar, la imaginación creativa y los nuevos usos de la infraestructura. Hacer parkour es un desafío, las tensiones entre quienes habitan un lugar y los traceurs emplazan otros cuerpos, esos que aun en sus diferencias se perciben, se señalan o se admiran en otras formas de expresarse, otros modos de recorrer el espacio y otros sentidos.

Dicen que todos los practicantes de $\mathrm{PK}$ son vándalos, el PK está mal visto, pero no ven lo real de nuestra disciplina, no es un deporte, es una disciplina, nosotros entrenamos la mente y cuerpo y le metemos el corazón a cada movimiento [...] la gente lo ve a uno y piensa "ya se va a escalar la casa" o que un policía pase y no nos diga: "bájese de ahí, marihuaneros", las cosas no son así; nosotros entrenamos porque queremos ser mejores cada día (EI-P4-GA).

Prácticas como el parkour expresan los cambios de sentido e intencionalidad que sufre el espacio a partir de su relación con el cuerpo. El bolardo 
más allá de constituir una barrera que impide al automóvil ocupar la acera, es un lugar de paso, una silla, un reto, un soporte del gesto motor y un motivante de la creatividad. El objeto sólido es superable en la fluidez corporal; vínculos que en muchos casos son contrarios a los propósitos de quienes han planificado y construido la ciudad desde la funcionalidad; por eso, la expresión "ser en el mundo" cobra especial importancia en esta práctica, "es vivir en movimiento intencional, siendo cuerpo consciente y comunicante. La existencia, a través de la Motricidad, es un continuo acto creativo; de un yo que implica hacer, saber, pensar, sentir, comunicar y querer" (Kon-traste y Trigo, 1999: 375).

En consecuencia, la ciudad como soporte, caótica y planificada en interacción con la práctica contribuye a gestar en el practicante un paisaje mental; en adelante, la escalera, la pared, la reja, el tubo y otros objetos más son referentes que le dan estructura a la mente y movimientos del traceur. Para los practicantes, la ciudad física es pensamiento en tanto los objetos que la configuran son fuente de reflexión y acción:

La ciudad me ha aportado los paisajes, las construcciones sólidas son las que le dan a uno el ánimo para realizar la práctica, uno ve una pared, ¡Uy, yo me la quiero trepar!, uno ve de pronto un bolardo, y también lo quiere pasar, pero analiza las múltiples formas para hacerlo (EI-P1-GC).

El parkour es una práctica que permite potenciar las capacidades, habilidades y destrezas físicas y cognitivas utilizando los movimientos y virtudes adaptativas del cuerpo como punto de partida y despliegue creativo.

La filosofía del PK se sustenta en la libertad del movimiento, la libertad que se encuentra en todo el entorno posible de observar, eso es lo bueno del PK, cada vez que uno llegue a un lugar uno quiere explorar su propia agilidad, su propio cuerpo (EG-P3-G2).

El parkour reconoce a la ciudad como un paisaje lleno de posibilidades y retos que va más allá de un lugar pasivo, funcional, vacío y carente de significados.

La ciudad, a la manera de la ficción de Calvino, tiene sus dimensiones (puede prevalecer una) la visible o la invisible, su lado legítimo o el ilegítimo, su lado terráneo o su dimensión subterránea, su dimensión triste o su dimensión alegre, su lado limpio o su lado sucio; ciudades abstractas, aéreas, sutiles, dobles, escondidas, utópicas (por descubrir), macabras, ellas, logran su condición (Moreno y Pulido, 2007: 11).

Dentro de este marco de referencias sensibles e imaginativas, los traceurs se encaminan hacia la búsqueda de la superación personal y la comprensión de sí mismos. Sus repertorios motrices van desarrollando una identidad que se proyecta en sus formas de vestir, hablar, relacionarse consigo mismo, con los demás y con el entorno, pero también por medio de 
emociones y experiencias comunicables, estetizadas, sensitivas y reflexivas abocadas a enfrentar el miedo propio y ajeno.

\section{El movimiento corporal y la imaginación como reclamos}

Una de las características más importantes en el parkour es la imaginación, no sólo como condición para su práctica, sino como proceso y resultado de las acciones de los traceurs. La imaginación siempre está en cualquier lugar, es una manifestación del pensamiento, pero también es un elemento emergente cuando el sujeto se relaciona con el espacio. La imaginación convierte a la ciudad en un parque, y aunque este contiene todo para la práctica, los traceurs reclaman espacios formales para posicionar el parkour como disciplina. Se trata de una exigencia como ciudadanos que reclaman un lugar, una espacialidad formal para sus cuerpos. "Es la imaginación quien permite expresar y comprender ideas. Ese poder combinador de la imaginación surge cuando se logra convocar adentro de la mente una imagen de manera significativa e inducir sentimientos profundos en su presencia" (Suarez, 2009: 171).

Exigir la construcción de estos escenarios hace parte de un conjunto de razones, acciones y procesos de pertenencia a la ciudad y de formación propios de la práctica que definen el sentido de la misma; por ejemplo, un lugar diseñado y construido para practicar parkour garantiza hasta cierto punto la integridad y cuidado del propio cuerpo y del ajeno; es decir, un proceso centrado en la corresponsabilidad. Por eso algunos practicantes señalan que la construcción de este tipo de escenarios constituye una invitación abierta a todos los ciudadanos simpatizantes de la práctica a vencer temores, ganar confianza y generar responsabilidad colectiva. Vale aclarar que la concentración de la práctica en lugares construidos para ella no implica límites a la imaginación y la diversidad, exigirlos hace parte de un reclamo ciudadano que invita a su reconocimiento.

La ciudad ofrece muchos lugares dada su naturaleza compacta, sólida y edificada, que opera a ritmo indefinible, la mayoría de las veces desde intereses grupales en los que las entidades estatales pierden valor; de ahí que los practicantes recurran a los espacios públicos y, en ocasiones, también privados para mostrar lo que son. Por ello, desde el parkour para el parkour y para la sociedad en general se propone vincular con mayor fuerza al sujeto con el espacio; por ejemplo, enseñando a los niños a trepar árboles o visitar parques, relaciones que fomentan y potencializan la creatividad y el aprendizaje.

La ciudad es un ente viviente, que puede respirar, que siente y se siente, por eso cada vez que hago uso de un obstáculo trato de cuidarlo, yo debo estar bien con la ciudad 
y ella lo estará conmigo, la ciudad es adaptación es nuestro escenario, así la conozco (EG-P4-GA).

El parkour se aprende familiarizándose con el espacio, es necesario recorrerlo, sentirlo, comprenderlo como posibilidad, pues cada cual imagina distintas formas de superar los objetos, de ahí que algunos traceurs asuman la ciudad como un parque donde se juega con los esquemas corporales, le quieren como a su propia casa. La ciudad es el hogar, pero también la libertad, particularmente para moverse y ser desde el cuerpo; por eso, cuando se reúnen con otros grupos, acuerdan la ruta precisando los movimientos y esquemas a realizar durante el desplazamiento sin olvidar que la regla general es velar por la seguridad del grupo; sin embargo, se juega con la variación que cada sujeto puede ofrecer al gesto y los obstáculos.

En consecuencia, el parkour es ampliamente exploratorio e imaginativo y visibiliza la diversidad de posibilidades que puede generarse en la calle; algunas de ellas, como es natural, generan tensiones momentáneas o pasajeras con otros habitantes de la ciudad como consecuencia del carácter nómada de la práctica. Estos conflictos suelen estar circunscritos al uso de espacios en los que se han determinado otras convenciones como: turismo, recreación, deporte, tránsito, entre otras.

\section{Cuerpo hogar}

Para el traceur la ciudad está viva, se requiere cuidar de ella como se cuidaría del propio cuerpo. Arruinar la infraestructura de la ciudad es una falta de cuidado de sí, razón por la cual se puede decir que la relación gesto intencional y espacio constituyen la tensión dialéctica fundante del parkour. Si bien es cierto que los objetos de la ciudad física se oponen, condicionan o armonizan con los movimientos, dimensiones o necesidades del cuerpo, también es claro que esto trae aparejadas otras relaciones que expresan el carácter inacabado de la ciudad, al igual que las posibilidades motrices e intencionales de los cuerpos.

En lo efímero de los trazos del cuerpo se redefine el sujeto; sin embargo, para el traceur nada es inmóvil, la nueva condición cuestiona el nuevo estado. La reflexión en torno al acontecimiento es la condición para lanzar una nueva trayectoria; es decir, una nueva forma de abordar y superar el obstáculo. Así, la armonía y fluidez del gesto motriz coincide con la preservación de la infraestructura; consentir implica dejar que la ciudad les forme. Simultáneamente, aparece un nuevo orden motriz que pronto muta; estar bien consigo mismo es consentir: dejar que la ciudad configure el cuerpo del traceur cuando este la recorre. 
Los traceurs describen y recorren la ciudad como analogía de sus propios cuerpos, interacción con el espacio que les permite la organización de sus movimientos. Sus relaciones reconocen que la ciudad no se puede ver de manera desarticulada; "la ciudad contemporánea acoge dentro de sus muros simbólicos diferentes tradiciones y orientaciones, se pierden en su interior y se mezclan diferentes culturas, experiencias vitales, angustias, temores y necesidades tanto individuales como sociales" (Hoyos, 2012: 64).

Las acciones de los traceurs constituyen nodos en los que confluyen el sujeto y la ciudad, porque ella permite la fluidez de la creatividad y las particularidades de la práctica y sus habitantes. Cabe resaltar que la construcción de la ciudad y otros territorios como analogía del cuerpo no es nueva. Múltiples grupos sociales asumen los referentes espaciales en clave funcional y homólogos al cuerpo orgánico; pero en estas ideas subyacen esquemas de relación propiamente sistémicos y ecológicos que articulan las formas de percibir, sentir y significar con sus propias representaciones corporales.

De este modo los traceurs reconocen a la ciudad como parte de sí mismos y de la práctica. Los referentes fijos, relacionales e imaginativos son parte de la vitalidad que los traceurs le asignan a la ciudad, sus espacios están integrados al propio cuerpo; cuidar la ciudad desde la práctica es un estado de bienestar propio.

Los traceurs ven y sienten el parkour de forma distinta según el contexto; se trata de una práctica en la cual los sentimientos de apego al espacio urbano son innegables, la ciudad es su casa, es el cuerpo que se habita, no existe distinción entre el norte y el sur, pues la ciudad los acoge en cualquier lugar: "la ciudad es nuestro lugar, es nuestra casa, así sea bien al sur o al norte es el lugar donde nos acogen y podemos hacer los movimientos, eso es para nosotros la ciudad" (EG-P2-GD).

El espacio de la ciudad les hace sentir bien, aunque en ocasiones la comprenden como contenedora de objetos-obstáculo y experiencias que les exige desarrollar un conjunto de habilidades adaptadas y adaptables a distintos lugares, y les obliga a cuidar del entorno.

Su filosofía se resume en la expresión "ser y durar", no sólo como sujeto sino como parte del contexto. En virtud de ello, la esencia del espacio depende de las acciones del traceur; esto significa una relación duradera por cuanto se requiere de preservar de la mejor manera los escenarios donde practican. En este sentido, el valor artístico de la práctica expuesto en la frase "el arte de desplazamiento" les lleva a realizar trazos suaves, estéticos, delicados, armónicos, reflexivos y fluidos que preservan los espacios. 


\section{Cambiando la percepción}

La manera de enfrentar el entorno es otra de las particularidades de la práctica; trasladarse de un punto A a un punto B de distintas formas, significa una fisura a la lógica lineal imperante en la planeación, diseño y construcción de la ciudad, no solo normativa sino formal. Para el traceur, la ciudad y sus obstáculos provocan miedo, pero incitan al riesgo, no hay límites, particularmente cuando el propósito es sortearlo con el cuerpo, pues un mismo obstáculo esconde múltiples formas de superarlo; en algunos casos, esos lugares que a los ojos de otros ciudadanos han perdido valor, para el traceur connotan posibilidades abiertas.

Las ruinas y espacios deteriorados de la ciudad también son de provecho para los practicantes, como lo declara uno de ellos: "había un hospital abandonado y nosotros nos subimos ahí, entramos porque el lugar es muy chévere, obviamente no era un lugar para entrar y ponerse hacer PK, entonces los vecinos llamaron a los policías y nos sacaron" (EG-P1-GC). De acuerdo con Rotawisky (2015: 200), "observar un entrenamiento grupal o individual sugiere lo intensa que es la relación que sus practicantes establecen con la infraestructura urbana, con la materialidad de la ciudad que frecuentemente es ignorada en los análisis sociales". La ciudad material constituida como depósito de comunidades (Massey, 2005) es inequívocamente inacabada, traceurs y espectadores o transeúntes se relocalizan, los primeros por el movimiento, los segundos por sus percepciones.

Con base en lo anterior, el aprovechamiento del entorno también se manifiesta en muchos de los objetos desechados en otras actividades: ruedas de tracto-camiones, cajas, tubos, estructuras de metal, entre otros que pueden ser reutilizados en virtud del espíritu adaptativo de la práctica; pues más allá de los acomodamientos del cuerpo a los objetos, estos cobran un nuevo valor al insertarlos en el paisaje, con otras finalidades distintas a las implícitas en sus diseños: "A veces encontramos cosas abandonadas en las esquinas, ruedas viejas, tablas, canecas o cosas así, las recogemos y las llevamos a parques o lotes para adecuar nuestros escenarios" (EI-P1-GD).

Los practicantes de parkour manifiestan no invadir los espacios públicos, los ocupan en breves fracciones de tiempo a medida que se desplazan, se adaptan fugazmente a ellos. El parkour es adaptación por autonomía, por voluntad y no solamente por estímulo. Para ello se recurre al método natural, moverse de forma fluida ante los obstáculos: "No sé escalar, voy a empezar subiendo un árbol” (EI-P4-GA). El método cobra sentido cuando la decisión y la voluntad se articulan con el espacio y su contenido; aprenderlo implica asumir un riesgo aun cuando esto se puede hacer mediante el cuidado de otro. 
Su lógica gira en torno al acomodamiento y armonización de las acciones y pensamientos; mediante estas manifestaciones el hombre ocupa su lugar, acciones concretas de relación del ser humano consigo mismo, con el mundo y con sus semejantes, una forma de relación que se da a través de su cuerpo y la acción humana que se encuentra cargada de significado (Pazos y Trigo, 2014).

Más allá de la devastación y deterioro de los lugares, el parkour se trata de una práctica de asimilación en la que el espacio se integra al esquema de movimientos del sujeto; así como surgen, los esquemas prontamente desaparecen sin dejar huella en los obstáculos; sin embargo, perdura en los traceurs como parte del espíritu de la práctica. En algunos casos, la responsabilidad con el espacio va más allá de su cuidado mediante acciones motrices suaves, fluidas, delicadas, pues el daño a la infraestructura conlleva un compromiso con el entorno, lo cual les obliga a repararle con sus propios recursos, mucho de ellos con dinero y otros veces con trabajo:

En algunas ocasiones hemos causado daño a espacios ya deteriorados, ladrillos que se caen, barandas mal puestas y cosas así. Cuando esto sucede recogemos dinero entre nosotros y luego las reparamos, todos aportamos algo; cuando no se tiene dinero, el aporte se hace con mano de obra (EG-P2-GD).

Aunque la práctica pretende cuidar de los espacios, es inevitable afectarlos, por eso los movimientos requieren el mayor grado de delicadeza, cualquier intento de enfrentar la ciudad por la fuerza es inviable, por tanto, la percepción de los objetos cambia, se tornan adversos pero necesarios, sin ellos no hay ninguna otra forma de moverse y recrear el paisaje urbano contenido en sus mentes. Un practicante dice: "Todo lo que encuentras en la ciudad es muy bueno, todo el inmobiliario se ve como un obstáculo, para mí, la ciudad es un parque" (EI-P5-GB). La ciudad se recrea y desvanece en los movimientos del cuerpo.

Son dos los ambientes en los cuales se hace evidente tal afirmación:

1) Los traceurs practican en espacios públicos, escenarios fijos que hacen parte del paisaje urbano. Las construcciones sólidas representan el reto, la ciudad es potenciadora, no por sí sola, sino por la relación que se establece con el sujeto; "la realidad se construye socialmente y siempre actúa por medio de la interpretación de los seres sociales en situaciones y espacios determinados histórico-socialmente" (Gergen, 1996: 74).

2) Los espacios sufren adecuaciones en las que se adicionan objetos. Estos lugares pueden ser públicos o privados, allí se construyen estructuras de soporte como cajones o barras, se amontonan ruedas de vehículos entre las que prefieren las de mayor tamaño, se entierran maderos, se adecuan las 
depresiones o cambios superficiales del suelo, entre otras acciones que configuran los campos de práctica.

\section{Consideraciones finales}

El parkour, contrario de lo que se puede creer, forma en sus practicantes un valioso sentido sobre el cuidado de la ciudad, no como una pieza de museo intocable, sino como lugar para vivir: necesario, amable, posible, pero también adverso, al cual es preciso confrontar para transformarlo. En este sentido, las acciones de los traceurs, a pesar de expresar emoción, delirio, diversidad, armonía, también comunican responsabilidad ante los espacios comunes y necesarios como condición para diálogo entre ciudadanos, no sólo como practicantes sino como comunicantes de la forma traceur; es decir, del moverse y vivir la ciudad. La fluidez de su hacer no es otra cosa que un mensaje que interroga las maneras de ordenar la ciudad, sus sistemas y lugares.

De acuerdo con lo anterior, el parkour aprovecha la estructura natural de cada lugar, su disposición y ubicación para convertirla en obstáculo. La mente del traceur demanda nuevos retos, no sólo en el orden de la destreza motriz, sino en función de la diversión, del gusto, el placer, la estética, la inspiración, la contemplación y el asombro; circunstancias por las cuales las posibilidades imaginativas desembocan en la construcción colectiva de una práctica y de un conjunto de técnicas corporales útiles al mundo humano.

El presente estudio pone en evidencia una nueva forma de lucha por el reconocimiento y valoración social; aquí el cuerpo sigue siendo protagonista, ahora por cuenta de un conjunto de técnicas de movimiento, situadas en los espacios públicos de la ciudad, imaginativas y a veces impredecibles, que reclaman un lugar sin violentar otros cuerpos, sin anularlos, sin caer en la violenta trampa del más fuerte, posesivo con el espacio, intimidante, invitando al cuidado del entorno, de la ciudad, de su propia casa. La práctica del parkour se la juega por afianzar los vínculos con el espacio y directamente con aquellos que lo ocupan.

Vale decir entonces que el parkour es una apuesta por formar otros sujetos a partir de la integración del espacio urbano en los esquemas corporales, nuevos vínculos con el mundo que reclaman espacios para madurar su espíritu. Los traceurs han construido otra ciudad en sus propios cuerpos, sus espacios están constituidos de movimiento y emociones que los remite al asfalto, al banco, al muro, como una forma de integrar cada entrada, cada salida, cada espacio urbano en sus maneras de moverse. 
Siguiendo las indicaciones de Viera y De Sousa (2016: 237), “se sugiere que se realicen investigaciones futuras que investiguen las representaciones que los practicantes poseen de su práctica”. La presente investigación pone en tensión una de las conclusiones a las que llegan los autores, cuando señalan "que las prácticas corporales corren el riesgo de tener sus principios valorativos distorsionados, lo que apunta a la necesidad de cautela con discursos mediáticos sesgados" (Viera y De Sousa, 2016: 237).

Tal distorsión parece corresponder a la perspectiva de observación que se abstiene de confrontar intencionalmente los comportamientos, valoraciones, ideas, relaciones y significados negativos delimitantes de la práctica, razón por la cual la información proveniente de personas o fuentes ajenas a esta puede ser contradictoria o veraz cuando se confronta con la información suministrada por los practicantes, de ahí la incongruencia y equivocada valoración, que no es otra cosa que un aspecto más de interés en las relaciones que se gestan cotidianamente y que, en aras de la objetividad, requieren ser objeto de análisis en los estudios de la práctica.

\section{Referencias}

Benjumea, Margarita (2010), La motricidad como dimensión humana, Colombia: Colección Léeme.

Carvalho, Rui y Pereira, Ana (2008), "Percursos alternativos: o parkour enquanto fenômeno subcultural”, en Revista Portuguesa de Ciência do Desporto, vol. 8, núm. 3, Portugal: Universidade Nova de Lisboa.

Coffey, Amanda y Atkonson, Paul (2003), Encontrar sentido a los datos cualitativos. Estrategias complementarias de investigación, Colombia: Universidad de Antioquia.

Cortés, Jhon, y Certuche, Johao (2014), "Parkour y ciudadanía: prácticas corporales alternativas en la ciudad”, en Memorias Congreso Asociación Latinoamericana de Estudios Socioculturales del Deporte (ALESDE) 2014, Colombia: ALESDE.

Eugeni, Joan (1991), Espacio, economia y sociedad, España: Siglo XXI.

Ferro, Lígia (2011), De la calle al mundo: configuraciones del graffiti y del parkour y campos de posibilidades urbanas, Portugal: Instituto Universitario de Lisboa.

Gergen, Kenneth (1996), Realidades y relaciones: Aproximaciones a la construcción social, España: Paidós.

Goffman, Erving (1989), La presentación de la persona en la vida cotidiana, Argentina: Amorrortu.

Gutiérrez, Rodolfo y Rosales, Diego (2011), "Parkour como medio motriz", en $9^{\circ}$ Congreso Argentino y 4 Latinoamericano de Educación Física y Ciencias, Argentina: Universidad Nacional de la Plata.

Hoyos, Jesús (2012), “La ciudad: espacio de construcción de sujeto”, en Informes psicológicos, vol. 12, núm. 1, Colombia: Universidad Pontificia Bolivariana.

Kon-traste, Equipo y Trigo, Eugenia (1999), Creatividad y motricidad, España: Inde. 
Leal, Néstor (1997), "El método fenomenológico: principios, momentos y reducciones”, en Revista Electrónica de Investigación Cientifica, Humanistica y Tecnológica, vol. 1, núm. 5, Venezuela: Universidad Nacional Abierta.

Le Breton, David (2002), Antropología del cuerpo y modernidad, Argentina: Nueva edición.

López, Daniela (2008), "El problema de la subjetividad en la explicación sociológica. Una mirada a partir del debate Schütz-Parsons", en Revista de Estudios Sociales, vol. 1, núm. 31, Colombia: Universidad de los Andes.

Massey, Dooren (2005), For space, Inglaterra: Sage.

Maturana, Humberto (1996), El sentido de lo humano, Chile: Dolmen.

Mclaren, Peter (1997), Pedagogía crítica y cultura depredadora. Politicas de oposición en la era posmoderna, España: Paidós.

Mèlich, Joan (1994), Del extraño al cómplice. La educación en la vida cotidiana, España: Anthropos.

Martínez, Jaime (2009), El movimiento humano, ciencia, competencias y estándares, ArmeniaColombia: Kinesis.

Moreira, Virginia (1998), “Técnicas de intervención fenomenológica en psicoterapia”, en, II Congreso Iberoamericano de Psicología, España: CD da Sección Psicología Clínica y Salud Mental.

Moreno, Laura (2009), "Enfermedad, cuerpo y corporeidad: una mirada antropológica”, en Gaceta de Medicina Mexicana, vol. 2, núm. 146, México: Universidad Nacional Autónoma de México.

Moreno, William y Pulido, Sandra (2007), Educación, cuerpo y ciudad. El cuerpo en las interacciones e instituciones sociales, Colombia: Funámbulos Editores.

Pazos José y Trigo, Eugenia (2014), “Motricidad Humana y gestión municipal”, en Estudios pedagógicos, vol. 11, núm. 1, Chile: Universidad Austral de Chile.

Ponty, Maurice (1994), Fenomenología de la percepción, España: Gráfica.

Redondo, Cristina (2011), “¿Qué es el Parkour?: origen. Habilidades: educación física en primaria como base para esta nueva modalidad deportiva”, en Revista Digital Innovación y Experiencia, núm. 38, España: Universidad de Granada.

Rotawisky, Jennifer (2015), “El arte del extravío: corporalidades y afectos en el Parkour”, en Revista Corpo-grafías: Estudios críticos de y desde los cuerpos, vol. 2, núm. 2, Colombia: Universidad Distrital Francisco José de Caldas.

Rotawisky, Jennifer (2013), “Parkour, cuerpos que trazan heterotopías urbanas”, en Revista Colombiana de Antropología, vol. 49, núm. 2, Colombia: Universidad Nacional de Colombia.

Scarnatto, Martin y Díaz, Julieta (2010), “De peatón a traceur en una diagonal. El Parkour en la ciudad de La Plata. Nuevas prácticas, patrimonios motrices y formas de socialidad", en IV Jornadas de Investigación en Educación Corporal, Argentina: Facultad de Humanidades y Ciencias de la Educación.

Sergio, Manuel (1999), Um corte epistemológico. Da educação física á motricidade humana, Portugal: Instituto Piaget.

Sergio, Manuel (1996), Epistemología de la motricidad humana, Portugal: Instituto Piaget.

Sennett, Richard (1987), Carne y piedra. El cuerpo y la ciudad en la civilización occidental, España: Alianza.

Silva, Valeria (2012), "Coletivos juvenis e Parkour no Brasil: percursos interculturais e Identidades”, en Tomo, vol. 5, núm. 21, Brasil: Universidade Federal de Sergipe. 
Strauss, Anselm y Corbin, Juliet (2002), Bases de la investigación cualitativa. Técnicas y procedimientos para desarrollar la teoría fundamentada, Colombia: Universidad de Antioquia.

Suarez, Mónica (2009), "La experiencia de la imaginación creadora como elemento primordial de la creación poética en la infancia”, en Civilizar, vol. 9, núm. 17, Colombia: Universidad Sergio Arboleda.

Trigo, Eugenia (2000), Fundamentos de la motricidad, España: Gymnos.

Trigo, Eugenia (1999), Creatividad y motricidad, España: Inde.

Vieira, Alessandra y De Sousa, Lilian (2016), "Parkour e valores morais: Ser forte para ser útil”, en Motrivivência, vol. 28, núm. 47, Brasil: Universidade Federal de Santa Catarina.

\section{Anexo}

\section{Tabla 1}

Grupos y fechas de realización de los encuentros de entrevista

\begin{tabular}{lllll}
\hline & Grupo A & Grupo B & Grupo C & Grupo D \\
\hline Encuentro 1 & febrero 9 de 2016 & marzo 16 de 2016 & mayo 5 de 2016 & julio 7 de 2016 \\
\hline Encuentro 2 & febrero 23 de 2016 & marzo 30 de 2016 & mayo 25 de 2016 & julio 27 de 2016 \\
\hline Encuentro 3 & marzo 9 de 2016 & abril 11 de 2016 & junio 14 de 2016 & agosto 10 de 2016 \\
\hline
\end{tabular}

Fuente: Elaboración propia.

\section{Tabla 2}

\section{Edades y años de experiencia de los practicantes}

\begin{tabular}{lcccccccccc}
\hline & P 1 & AE & P 2 & AE & P 3 & AE & P 4 & AE & P 5 & AE \\
\hline Grupo A & 18 & 6 & 21 & 6 & 22 & 6 & $\underline{29}$ & 12 & 20 & 5 \\
\hline Grupo B & 25 & 5 & 25 & 5 & 23 & 6 & 20 & 5 & $\underline{32}$ & 7 \\
\hline Grupo C & $\underline{23}$ & 5 & 20 & 6 & 19 & 5 & 19 & 5 & 18 & 7 \\
\hline Grupo D & $\underline{34}$ & 14 & 30 & 6 & 23 & 5 & 20 & 6 & 21 & 9 \\
\hline $\begin{array}{l}\text { Entrevistados } \\
\text { individuales }\end{array}$ & 23 & 4 & 29 & 14 & 26 & 9 & 30 & 7 & 29 & 10 \\
\hline
\end{tabular}

Fuente: Elaboración propia. 
Jhon Carlos Cortés-Murillo. Maestrando en Estudios Sociales, Universidad Pedagógica Nacional de Colombia. Antropólogo, Universidad Nacional de Colombia. Licenciado en Educación Física, Universidad Pedagógica Nacional de Colombia. Director de la Línea de investigación Motricidad y Desarrollo Humano, en Corporación Universitaria CENDA. Docente e investigador en Corporación Universitaria CENDA, Colombia. Líneas de investigación: cuerpo y posmodernidad, historia de la educación física en Colombia, espacio y cotidianidad, violencia escolar y educación física. Publicaciones recientes: Cortés, Jhon y Certuche, Johao (2017), "Éramos niños y queríamos correr y jugar. La educación física más allá de las normas y los muros de la escuela”, en Lúdica Pedagógica, vol. 1, núm. 24, Colombia: Universidad Pedagógica Nacional; Cortés, Jhon y Certuche, Johao (2015), "Sobre el hecho de ser ciudad y ciudadanía. Condiciones espaciales de práctica en educación física”, en Revista Horizontes Pedagógicos, vol. 17, núm. 2, Colombia: Universidad Iberoamericana.

Ita del Pilar Perea-Baena. Magister en Administración de empresas con especialidad en Dirección de proyectos, Universidad Viña del Mar, Chile. Licenciada en Educación básica con énfasis en educación física, recreación y deporte. Docente-investigador en la línea Motricidad y Desarrollo humano, en Corporación Universitaria CENDA, Colombia. Líneas de investigación: didáctica de la educación física, deporte social comunitario, deporte, sociedad y cultura.

Johan Sarmiento-Rojas. Maestrando en Ciencias del Deporte UDCA. Profesional en Ciencias del deporte y educación física, Universidad Cundinamarca. Aspirante a magister en Ciencias del deporte de la Universidad de Ciencias Aplicadas y Ambientales. Docente-investigador en la línea Motricidad y Desarrollo humano, en Corporación Universitaria CENDA, Colombia. Líneas de investigación: deporte y movimiento, tecnologías aplicadas al deporte. 